\title{
Simulation of the Effect of Plasma Flows in DIII-D JET, and JT-60U ${ }^{1}$
}

G.D. Porter, T.D. Rognlien, M.E. Rensink, A. Loarte, N. Asakura, H. Takenaga, G. Matthews

\section{May 17, 2002}

Lawrence Livermore National Laboratory

This article was submitted to $15^{\text {th }}$ International Conference on Plasma Surface Interactions (PSI) in Controlled Fusion Devices, Gifu, Japan, May 27-31, 2002

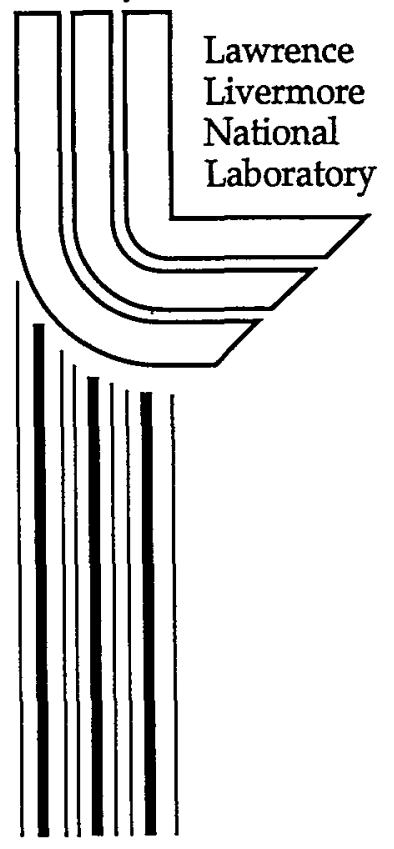




\section{DISCLAIMER}

This document was prepared as an account of work sponsored by an agency of the United States Government. Neither the United States Government nor the University of California nor any of their employees, makes any warranty, express or implied, or assumes any legal liability or responsibility for the accuracy, completeness, or usefulness of any information, apparatus, product, or process disclosed, or represents that its use would not infringe privately owned rights. Reference herein to any specific commercial product, process, or service by trade name, trademark, manufacturer, or otherwise, does not necessarily constitute or imply its endorsement, recommendation, or favoring by the United States Government or the University of California. The views and opinions of authors expressed herein do not necessarily state or reflect those of the United States Government or the University of California, and shall not be used for advertising or product endorsement purposes.

This is a preprint of a paper intended for publication in a journal or proceedings. Since changes may be made before publication, this preprint is made available with the understanding that it will not be cited or reproduced without the permission of the author.

This report has been reproduced directly from the best available copy.

Available electronically at http://www.doe.gov/bridge

Available for a processing fee to U.S. Department of Energy and its contractors in paper from

U.S. Department of Energy

Office of Scientific and Technical Information

$$
\text { P.O. Box } 62
$$

Oak Ridge, TN 37831-0062

Telephone: (865) 576-8401

Facsimile: (865) 576-5728

E-mail: reports@adonis.osti.gov

Available for the sale to the public from

U.S. Department of Commerce

National Technical Information Service

5285 Port Royal Road

Springfield, VA 22161

Telephone: (800) 553-6847

Facsimile: (703) 605-6900

E-mail: orders@ntis.fedworld.gov

Online ordering: http://www.ntis.gov/ordering.htm

OR

Lawrence Livermore National Laboratory

Technical Information Department's Digital Library

http://www.llnl.gov/tid/Library.html 


\section{Simulation of the effect of plasma flows in DIII-D, JET, and JT-60U 1}

G D Porter, T D Rognlien ${ }^{1}, \mathrm{M}$ E Rensink ${ }^{1}$, A Loarte ${ }^{2}$, N Asakura ${ }^{3}, \mathrm{H}_{\text {Takenaga }}{ }^{3}$, G Matthews ${ }^{4}$, and contributors to the EFDA-JET work programme

${ }^{1}$ Lawrence Livermore National Laboratory, Livermore CA, USA

${ }^{2}$ EFDA-CSU, Max-Planck-Institut für Plasmaphysik, D-85748 Garching, Germany

${ }^{3}$ Japan Atomic Energy Research Institute, Naka-machi, Naka-gun, Ibaraki-ken,311-01, Japan

${ }^{4}$ EURATOM/UKAEA Fusion Association, Culham, Abingdon, Oxon. OX14 3DB, UK

\section{Abstract}

The results of $2 \mathrm{D}$ fluid plasma simulations of the ion flow in the boundary plasma of DIII-D, JET, and JT-60U are reported. The model includes the effects of drifts and of impurity radiation using a multi-species model of intrinsic carbon impurities. Drift effects are important in determining the primary and impurity ion fluxes in the SOL and the private region, with ExB drifts dominant. Siniulated parallel velocities are consisteint wirh. experimental measurement on the high fiedd side of JT-60U, where the parallel flow is determined by ion sources. Simulated parallel velocities are significantly less than seen in experiment at the outer midplane of IT-60U, and at the top of JET where the flow is sensitive to poloidal variations of the turbulence driven transport, suggesting the velocity in these regions is determined by transport. Parallel flows are reversed by changing the direction of the ion $\nabla \mathrm{B}$ drift relative to the $\mathrm{X}$-point. $(150$ words $)$

\section{Introduction}

Understanding the flow of plasma in the scrape-off layer (SOL) of a tokamak device is very important. The drag between ion species flowing at different rates is an important force

\footnotetext{
${ }^{1}$ This work was performed under the auspices of the U.S. Department of Energy by the University of California, Lawrence Livermore National Laboratory under Contract No. W-7405-Eng-48.
} 
which controls the flow of impurities from the divertor regions to the closed field lines where they can dilute the plasma and reduce the fusion yield. The flow of carbon, in devices with carbon plasma facing components, determines the location of net deposition of carbon, and concomitant accumulation of hydrogen isotopes. This is particularly relevant for design of future devices which will operate with tritium because of the seriousness of the tritium retention expected with the use of carbon. Flow of primary ions in the SOL dominates the physics of recycling, and hence controls the nature of fueling of the core plasma.

Understanding the controlling factors for plasma flow in the SOL can therefore assist in efficient design of divertor structures to optimize a broad range of plasma physics issues.

The results of simulation of the plasma flow in three devices, DIII-D, JET, and JT-60U, are reported here. The flow is modeled using the $2 \mathrm{D}$ fluid plasma code UEDGE[1]. We describe the nature of the discharges modeled for each device in Section 2, and give a brief description of the physics of the models used for simulation in Section 3. The simulation results are described in Section 4, and we conclude with a brief summary of the results in Section 5.

\section{Description of discharges modeled}

Four discharges have been selected for simulation in this study; one each from JET and IT-60U, and two from DIII-D. These discharges are all in either Ohmic or low confinement mode (L-mode). They were selected primarily because of the existence of significant diagnostic information about the nature of the boundary piasma. The JT-60U discharge is a lower single null (LSN) plasma with 4.5 MW neutral bearn heating. The ion $\nabla \mathrm{B}$ drift is toward the $\mathrm{X}$-point for this configuration. This was one of a series of discharges in which the . plasma density was varied and the flow pattern was determined with the use of insertable Mach probes at three poloidal locations. The JET discharge was also a LSN configuration with the ion $\nabla \mathrm{B}$ drift toward the X-point. The JET discharge is Ohmically heated with a total 
heating power of 1.8 MW. The diagnostic set applied to this discharge includes an insertable Mach probe near the top of the plasma. Two DIII-D discharges were selected for this study, one LSN and the second an upper single null (USN) configuration. Since the field direction was held fixed, this provides data with the ion $\nabla \mathrm{B}$ drift both towards and away from the X-point. Both DII-D discharges are in L-mode with 1.8 MW neutral beam heating.

\section{Description of simulation model}

The behavior of the boundary plasma for the four discharges described here has been examined using the 2D fluid plasma code UEDGE. This code solves the Braginskii fluid equations for particle continuity, electron and ion thermal transport, ion parallel momentum, and electrostatic potential. Kinetic effects are included through flux limits for all parameters. The behavior of neutrals is modeled using a Navier-Stokes fluid model which includes the effect of ion/neutral collisions on parallel momentum. We include the effect of intrinsic carbon sputtered from the plasma facing surfaces using a multi-species impurity model which soives parallel transport using a force balance model.[2] Cross field transport of all parameters is assumed to be dominated by turbulence, and is modeled with a simple specification of cross field diffusivities. This model has been extensively compared with data from the DII-D device.[3] The amplitude of the diffusivities are determined by matching the simulated profiles of density and temperature with measurement in the vicinity of the outer midplane of the main plasma. In addition to the usual physics, we include the effect of plasma drifts in the results reported here, using the model described by Rognlien.[1,4] The radial gradient scale length of plasma density and temperatures is set to be $5 \mathrm{~cm}$ at the equivalent of a wall within the calculational domain, i.e. at the outermost flux surface, and the extreme of the private flux region. The ion flux across these surfaces are assumed to recycle as neutrals. The ion flux to the divertor plates is also assumed to recycle as neutrals. 
The primary ion density on the innermost flux surface (the $94 \%$ poloidal flux on closed field lines) is used as a boundary condition, as is the power. Particle throughput is determined by specifying an albedo for the neutral flux to the plasma facing surfaces. The albedo has been selected consistent with the best information available on the pumping capability of each device. Thus, for example, the neutral albedo used in JET is unity everywhere expect a narrow region on the vertical walls of the inner and outer divertors, corresponding to the location of the pumping ducts on that device.

\section{Results}

The radial profiles of the simulated poloidal flux of primary ions at two poloidal locations for a JT-60U simulation are shown in Figure 1. The simulation results obtained just above the high field side of the X-point, and just below the low field side of the X-point are shown. The profile is mapped back to the outer midplane to avoid confusion arising from the poloidal variation of the magnetic flux expansion. Note, however, that fluxes shown for $\Delta R_{\text {sep }}<0$ lie on the closed files line at the high field side, and in the private flux region for the low field side. The total poloidal flux is shown in red; the projection of the parallel flux onto the poloidal: plane in green; and the projection of the cross field flux from $\mathrm{E} \times \mathrm{B}$ in dark blue. The flux arising from the projection of the ion $\nabla \mathrm{B}$ drift onto the poloidal plane, shown in cyan, is insignificant at all poloidal positions. In general, the contribution of the flux from $E \times B$ and that from parallel flows are comparable. Hence the drift terms are important in determining the total ion flow. The sheared poloidal flux on the closed flux surfaces, indicated by the simulation at the high field side, is relevant to understanding $\mathrm{H}$-mode pedestal physics. The large poloidal flux shown in the PF region on the low field side, indicates a large flow of primary ions from the outer divertor to the inner divertor driven by the large radial electric field associated with steep radial electron temperature gradients near the separatrix below the 
$\mathrm{X}$-point. This large poloidal flux is also seen in the carbon ions, as shown in Figure 2, and indicates a flow of carbon ions to the inner divertor. This may be important in understanding the profile of carbon redeposition, and concomitant hydrogen (tritium) retention. The large poloidal flow of carbon in the private region is not seen in all simulations. In particular, some simulations of JET with low pumping of the primary fuel produced solutions near detachment. In this case, the electron temperature in the outer divertor region is reduced, leading to much lower radial electric fields, and hence small poloidal flux from the $\mathrm{E} \times \mathrm{B}$ drift term.

The Mach probes used in experiment measure the parallel velocity, and thus only determine a portion of the total ion flow. However, it is instructive to compare the simulated parallel Mach numbers with those measured in experiment, as a means of assessing the consistency of the simulation with experiment. The radial profiles of the simulated parallel Mach number are compared with experimental results measured at the high field side of JT-60U, and at the plasma top in JET, in Figure 3. Consider first the JT-60U high field side data. Although the radial profile is different, the UEDGE simulation accurately reproduces the maximum parallei Mach number of 0.5 , toward the inner divertor. The increase in the Mach number seen in the experiment outside the $4 \mathrm{~cm}$ flux surface arises because of acceleration of the ions to Mach 1 in the sheath near the divertor plate. The initial part of this is also seen in the simulation, but is not as dramatic as seen in the experiment, indicating the code diagnostic doesn't accurately reproduce the geometry of the experiment due in part to the relatively coarse poloidal mesh above the $\mathrm{X}$-point. The simulations indicate the parallel velocity in the SOL increases as the upstream plasma density increases, as seen in experiment.[5] The parallel flow on the high field side of the X-point are driven largely by ionization of neutrals escaping from the inner divertor region and penetrating up the inner post, i.e. the flow is driven by atomic physics and neutral transport. 
The simulation of the JET data indicates that the UEDGE code underestimates the ion parallel velocity at the top of the plasma by at least a factor of 2. Similar discrepancies are seen in simulations of the JT-60U data from the outer midplane and the low field side of the $\mathrm{X}$-point. The JET experiment consistently measures a large parallel velocity at the top of the plasma. The flow is typically toward the inner divertor, as seen in both the data and simulation in Figure 3(b). Simulation of two DII-D discharges indicates that the parallel velocity in this region is reversed when the ion $\nabla \mathrm{B}$ drift direction is changed from toward to away from the $\mathrm{X}$-point. The simulated parallel flow velocity at the top of the JET plasma is relatively insensitive to variations in the divertor pumping rates, and the level of gas puffing at the top of the plasma. This suggests that the simulated flow is not driven by atomic physics since those processes are predominately restricted to the divertor region with large recycling neutral densities. The simulated flow velocity is seen to increase when the spatially constant cross field diffusivities used in Figure 3 are replaced with diffusivities which have a ballooning character. Specifically, the flow velocity increased by about $20 \%$ when diffusivities which varied inversely as the square of the local magnetic field were used. This suggests that the large measured flow rates may arise from details of the turbulence driven . transport, i.e. it is driven by transport, not atomic physics.

\section{Summary}

Simulation of the boundary plasma in three tokamaks indicate that drift effects play a significant role in determining the flow of plasma in the SOL. The models used in this study included the effects of plasma drifts, and of impurity radiation arising from carbon sputtered from the plasma facing components of the devices. A large ion flux of both primary and impurity ions is seen in the PF region near the separatrix in devices with attached plasmas in the outer divertor. This flow is from the outer to inner divertor regions for plasma with the 
ion $\nabla \mathrm{B}$ drift toward the $\mathrm{X}$-point. This flow is driven by large radial electric field associated

with steep electron temperature gradients between the PF and SOL regions. These flows may play a role in determining the spatial profile of carbon deposition and concomitant co-deposition of hydrogen isotopes. The simulated parallel flow velocities are consistent with measurement in poloidal regions dominated by large ionization sources of recycling neutrals. The simulated parallel velocities in regions away from these ionization regions are significantly lower than measured. It is postulated that the parallel flow in these regions is determined by plasma transport processes which are poorly modeled with the simple transport model assumed in the results reported here.

\section{References}

1 T D Rognlien, G D Porter, and D D Ryutov, J. Nucl. Mat. 266-269, 654 (1999).

2 G.R. Smith, P.N. Brown, R.B. Campbell et al., J. Nucl. Mat. 220-222, 1024 (1995).

$3 \quad$ G D Porter, R Isler, J Boedo et al., Phys. Plasma 7 (9), 3663 (2000).

$4 \quad$ T D Rognlien, D D Ryutov, and N Mattor, Czech. J. Phys. 48 (S2), 201 (1998).

5 N Asakura, $H$ Takenaga, $K$ Shimizu et al., presented at the IAEA TCM on Divertor .. Concepts, Aix-en-Provence, 2001 (unpublished).

\section{Figures}




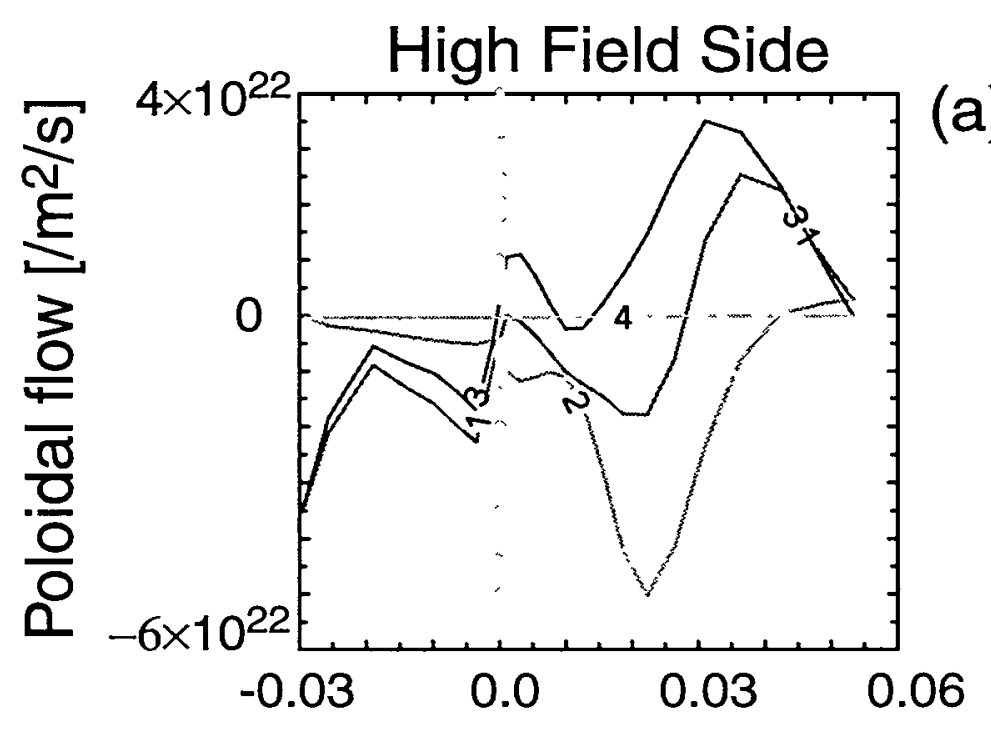

(a)

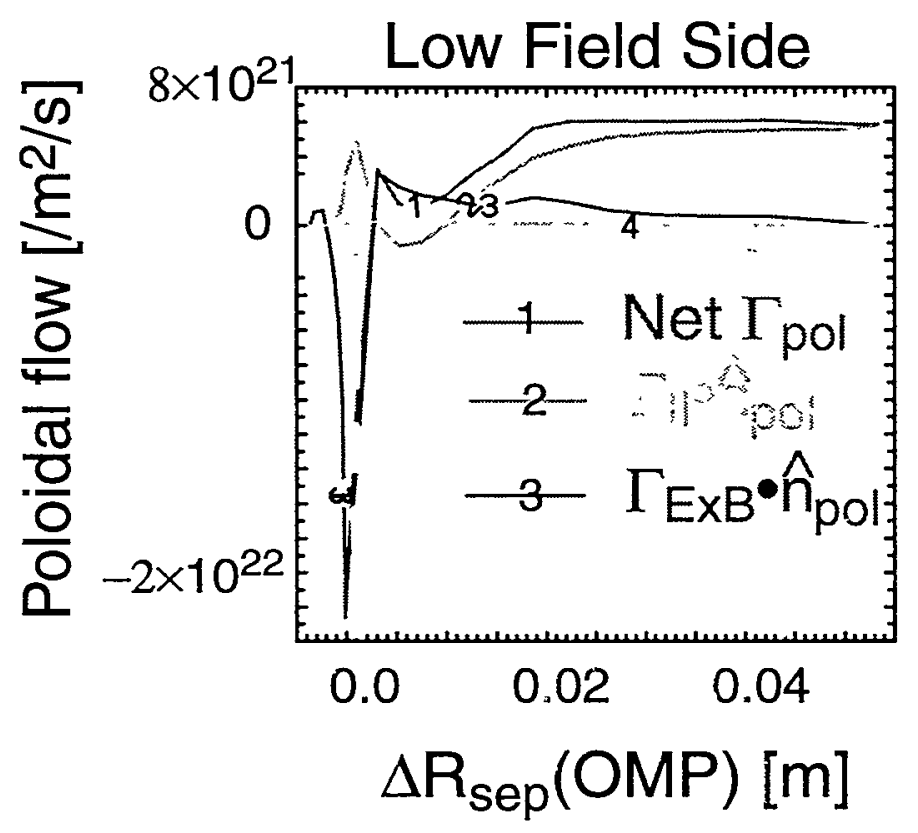

(b)

Figure 1 Components of the poloidal mass flow for a JT-60U simulation. The red (1) curves show the net flow; the green (2) curves show the projection of the parallel flow onto a poloidal plane; and the blue (3) curves show the projection of the flow from EXB onto the poloidal plane. The top panel (a) is obtained just above the high field side of the X-point, and the lower panel (b) just below the low field side of the X-point. 


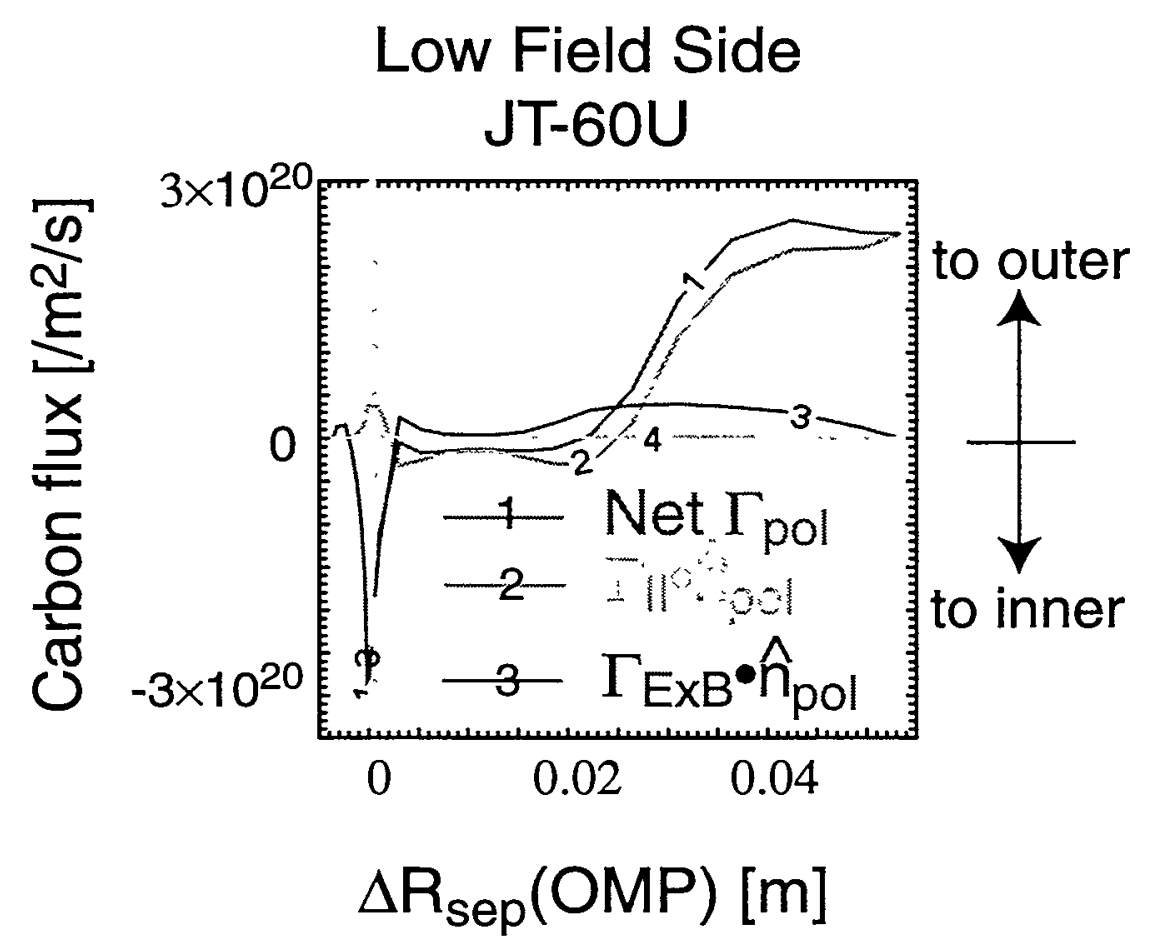

Figure 2 Poloidal flux of carbon simulated just below the low field side of the X-point for JT-60U. 

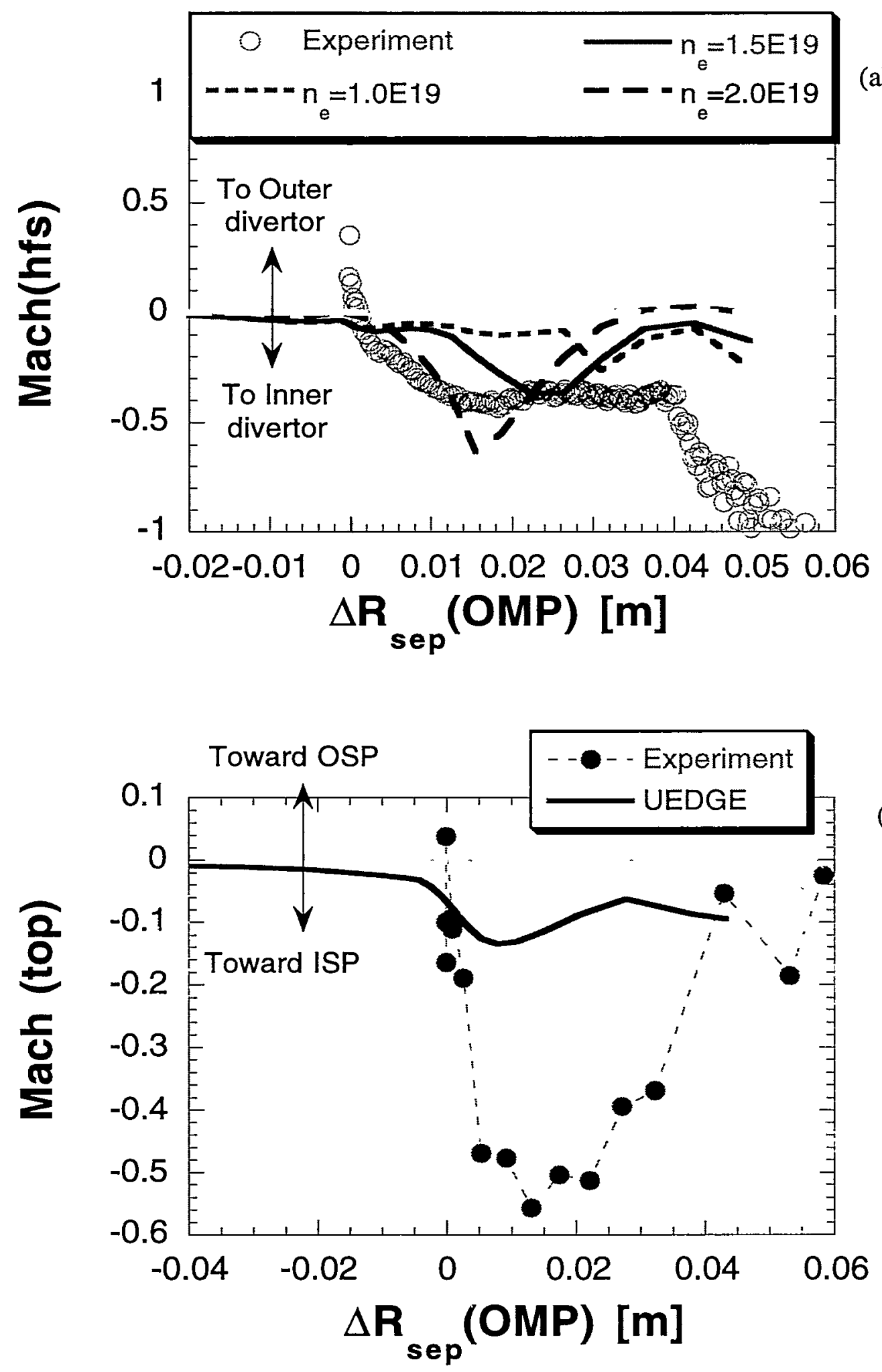

Figure 3 Comparison of simulated parallel Mach number on the high field side of the X-point of JT$60 U$ with data from a retractable probe(a), and simulation of the parallel Mach number with data obtained at the top of the JET plasma with data $(b)$. 